\title{
NOUVELle
}

\section{Construction d'un embryon de vertébré à partir de deux gradients de morphogènes}

Bernard Thisse, Christine Thisse
Department of Cell Biology, University of Virginia, Charlottesville, Virginia, દ́tats-Unis. christhisse@virginia.edu
> Le développement de l'embryon de vertébré dépend de processus moléculaires et cellulaires complexes qui instruisent progressivement les cellules embryonnaires, leur conférant leur identité et contrôlant leur comportement. Durant les deux dernières décennies, de nombreuses activités géniques ont été identifiées comme essentielles au développement de l'embryon de vertébré $[1,2]$. Néanmoins, les conditions minimales requises pour instruire les cellules embryonnaires pluripotentes et les organiser en différents tissus et organes n'avaient pas été établies. Nous avons relevé le défi et avons recherché les conditions expérimentales permettant de construire un embryon complet en instruisant des cellules d'embryon précoce de poisson zèbre (Danio rerio) à partir de combinaison de signaux morphogéniques.

\section{Un peu d'histoire}

Les premières tentatives ayant pour but de construire un embryon de vertébré datent d'il y a 90 ans lorsque Hilde Mangold, étudiante dans le laboratoire de Hans Spemann, transplanta la lèvre blastoporale dorsale d'une gastrula de triton (Triturus taeniatus) dans la partie ventrale d'une gastrula d'une autre espèce de triton (Triturus cristatus, dont les cellules ne sont pas pigmentées) [3]. II en résulta la formation, au site de la greffe, d'un axe embryonnaire secondaire qui comprenait des cellules pigmentées du greffon (provenant de Triturus taeniatus) ainsi que des cellules de l'hôte (Triturus cristatus, non pigmentées). À cause de sa capacité à organiser les cellules de l'hôte, la lèvre blastoporale dorsale du donneur fut définie comme un tissu organisateur et est connue depuis lors sous le nom de « centre organisateur de Spemann ». La nature moléculaire de ce centre organisateur dorsal a été identifiée durant les deux dernières décennies [4], et les éléments essentiels de son activité correspondent à des facteurs sécrétés par la lèvre blastoporale dorsale, qui agissent comme antagonistes de morphogènes ventraux, et conduisent à l'établissement d'un gradient d'activité ventro-dorsal de ces morphogènes (Figure 1A). Dans l'expérience pionnière réalisée par Spemann et Mangold, les facteurs dorsaux sécrétés par le greffon vont inhiber l'activité des morphogènes ventraux présents au site de la greffe et générer un nouveau gradient, qui récapitule dans le territoire ventral, et comme une image en miroir, le gradient d'activité morphogénique présent chez l'embryon sauvage dans ses partie latérales et dorsales (Figure 1B). C'est donc par une modulation des signaux présents dans la région marginale ventrale de l'embryon que les greffes du centre organisateur de Spemann conduisent à l'organisation d'un axe embryonnaire secondaire.

Cependant, lorsque ce centre organisateur est greffé dans un environnement neutre, tel que le pôle animal d'une blastula de poisson zèbre, nous avons montré qu'il ne présente qu'une activité réduite et ne conduit qu'à la formation de tissus mésendodermiques axiaux tels que la notochorde [5]. Ce résultat suggérait que l'activité (ou les activités) organisatrice(s) de l'embryon s'étendai(en)t au-delà de ce centre organisateur dorsal.

$\varepsilon n$ greffant différentes portions de la marge embryonnaire de blastula ou de celle de gastrula de poisson zèbre au pôle animal d'un embryon hôte au stade blastula, nous avons découvert que la marge embryonnaire ventrale agit comme un organisateur caudal [5], alors que les régions latérales et dorso-latérales de la marge embryonnaire organisent respectivement le tronc et la partie céphalique postérieure [6]. Ainsi, les activités organisatrices de l'embryon ne sont pas, contrairement au dogme en vigueur, restreintes au centre organisateur de Spemann, mais sont en fait distribuées tout le long de la marge embryonnaire et forment un continuum du territoire dorsal jusqu'au territoire ventral [6].

\section{Deux gradients de morphogènes, BMP et Nodal, définissent} les conditions minimales pour organiser un axe embryonnaire complet

La marge embryonnaire de l'embryon est le site d'expression de nombreux facteurs inducteurs. C'est en particulier le cas des facteurs Nodal et BMP (bone morphogenic protein). La voie de signalisation BMP est active ventralement et son activité décroît progressivement en direction de la marge dorsale, alors que l'activité Nodal est présente dans toutes les cellules marginales, mais est plus forte à la marge dorsale (Figure 1C). II existe ainsi une variation continue du rapport des activités BMP/Nodal depuis les territoires ventraux où ce rapport est élevé, vers les territoires dorsaux où il est nul (Figure 1C). 


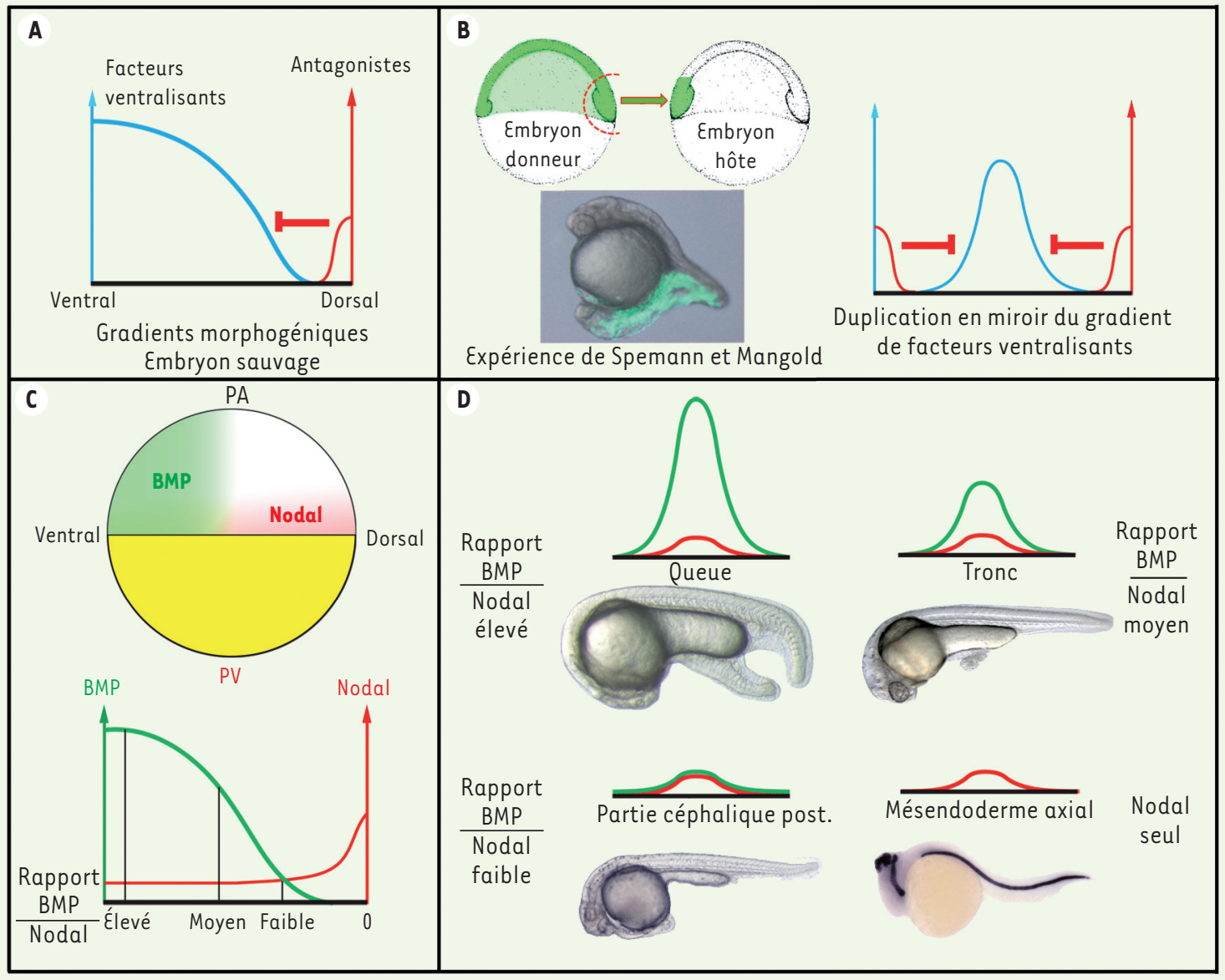

Figure 1. L'organisation de l'axe embryonnaire dépend du rapport d'activité des voies de signalisation BMP et Nodal. A. Gradients morphogéniques chez l'embryon sauvage. B. L'expérience de Spemann et Mangold (illustrée ici chez le poisson zèbre) entraîne l'expression ectopique, en position ventrale, des facteurs antagonistes dorsaux. Ceci conduit à une duplication en miroir du gradient des facteurs ventralisants présents chez l'embryon sauvage. $C$. Le rapport des activités BMP/Nodal varie continuellement de la marge ventrale à la marge dorsale de l'embryon sauvage. Les territoires où BMP et Nodal sont actifs sont présentés pour un embryon au stade blastula tardive. PA : pôle animal, PV : pôle végétatif. $D$. La stimulation des cellules du pôle animal avec différents rapports BMP/Nodal conduit à l'induction des différentes parties de l'axe embryonnaire. Un rapport BMP/ Nodal élevé induit la formation d'une queue ectopique [5]. Un rapport moyen induit la formation de structures troncales [6], un rapport faible induit la formation de la tête postérieure [6], tandis que l'injection de Nodal seul induit seulement la formation de structures mésendodermiques axiales $[5,6,8]$ telles que la notochorde révélée ici par hybridation in situ avec un gène spécifiquement exprimé dans cet organe.

Nous avons montré que l'injection, dans un blastomère du pôle animal au stade 128 cellules, de mélanges d'ARN messagers codant pour BMP et Nodal résulte en la formation de structures ectopiques identiques à celles qui sont induites par les greffons issus des différentes régions de la marge embryonnaire (Figure 1D). L'injection d'ARNm dans un rapport BMP/ Nodal élevé (25 fois plus d'ARNm BMP que d'ARNm Nodal) conduit à la formation de queues ectopiques, alors que des rapports plus faibles (quantité d'ARNm
BMP $5 x$ ou $1 x$ supérieure à l'ARNm Nodal) résultent en l'induction de structures troncales ou de parties céphaliques postérieures [6]. Enfin, l'injection d'ARNm Nodal seul conduit à la formation de mésendoderme axial, mimant ainsi l'activité du centre organisateur de Spemann lorsque ce dernier est greffé au pôle animal de l'embryon.

Sur la base de ces observations, nous avons postulé que deux gradients opposés de Nodal et de BMP qui permettraient de créer une variation continue du rapport d'activité BMP/Nodal devraient être suffisants pour générer l'ensemble des activités organisatrices de la marge embryonnaire, et donc pour instruire des cellules embryonnaires pluripotentes et les conduire à former un axe embryonnaire complet.

Pour tester cette hypothèse, nous avons ainsi généré deux clones, I'un sécrétant BMP et l'autre Nodal, par injection d'ARNm codant pour ces facteurs dans deux blastomères distincts du pôle animal d'un embryon au stade 128 cellules 

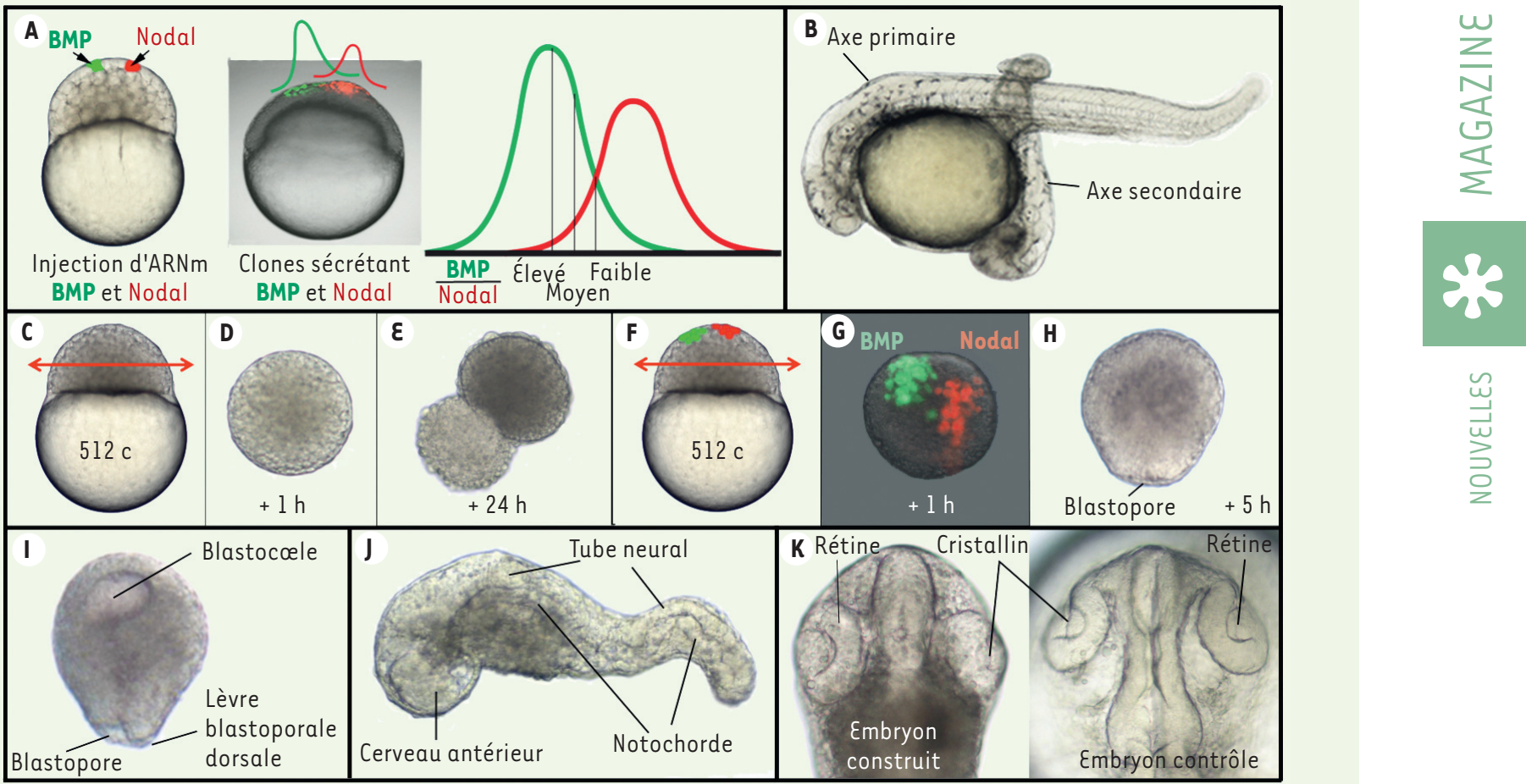

Figure 2. Construction, in vivo et in vitro, $d^{\prime}$ 'un axe embryonnaire complet à partir de deux gradients de morphogènes, BMP et Nodal, instruisant les cellules naïves du pôle animal d'un embryon de poisson zèbre. A. La création expérimentale de deux gradients opposés de BMP et de Nodal (par injection de leurs ARN respectifs dans des blastomères séparés) récapitule au pôle animal de l'embryon la variation graduelle du rapport entre les activités des voies de signalisation BMP et Nodal observée dans la région marginale d'un embryon sauvage. Ceci conduit (B) à l'organisation d'un axe secondaire complet à partir des cellules du pôle animal [7]. C. Explantation au stade 512 cellules du territoire animal d'un embryon de poisson zèbre (double flèche rouge). D. Placé en milieu de culture, l'explant devient sphérique au bout d'une heure et les cellules superficielles se différencient en cellules de la couche enveloppante (précurseur du périderme) [7]. Après $24 \mathrm{~h},(\varepsilon)$ la couche péridermique est rompue suite à la prolifération et la croissance des cellules embryonnaires. En absence de stimulation, aucun tissu ou organe n'est observé [7]. $F_{*}$ Explantation de la région du pôle animal après injection dans deux blastomères séparés d'un mélange d'ARNm BMP et de fluorescéine-dextran d'une part, et d'un mélange d'ARNm Nodal et de rhodamine-dextran d'autre part. G. Après 1 h en milieu de culture, l'explant est sphérique et la position des clones BMP (vert) et Nodal (rouge) est visualisée par les fluorescences de la fluorescéine et de la rhodamine [7]. H. À 5 h de développment, l'explant instruit par les gradients morphogéniques BMP et Nodal forme un blastopore et gastrule [7]. I. L'organisation de cette gastrula est très similaire à celle d'une gastrula d'embryon d'amphibien avec une cavité blastocœlienne en position animale et une asymétrie du blastopore, avec une lèvre blastoporale dorsale plus épaisse [7]. J. Après $24 \mathrm{~h}$ en milieu de culture, les explants instruits forment des embryoüdes très similaires aux embryons sauvages et dans lesquels on peut distinguer par exemple un cerveau antérieur, un tronc contenant un tube neural et une notochorde [7]. $\boldsymbol{K}$. Vue dorsale de la partie céphalique d'un embryoïde construit par instruction d'explant du pôle animal. Cette partie céphalique est très similaire à celle d'un embryon sauvage et est caractérisée notamment par la présence de deux yeux (comprenant rétine et cristallin) séparés par du tissu céphalique antérieur.

(Figure 2A). Nous avons observé la formation d'axes embryonnaires secondaires formés de tissus et d'organes identiques à ceux de l'embryon sauvage [7]. Dans la plupart des cas, les territoires antérieurs des axes primaire et secondaire sont fusionnés car il existe une compétition entre ces deux axes pour le recrutement des cellules du pôle animal. Toutefois, dans certains cas, les deux axes ne fusionnent pas, et l'axe embryonnaire secondaire induit au pôle animal présente alors une tête, un tronc et une queue séparés de l'axe embryonnaire primaire (Figure 1B). Ces embryons secondaires possèdent leur propre cœur, ont une symétrie bilatérale, deux yeux et deux oreilles, et on observe des contractions myotomales indiquant la présence d'un système nerveux fonctionnel.

\section{Construction d'un embryon in vitro}

Les gradients opposés de Nodal et BMP peuvent non seulement conduire à la formation d'un axe embryonnaire complet et indépendant de l'axe embryonnaire primaire lorsque ces derniers sont générés expérimentalement au pôle animal d'un embryon, mais ils peuvent également instruire des cellules embryonnaires pluripotentes in vitro. 
Les cellules de la région animale d'une jeune blastula, explantées et placées dans un milieu de culture, forment rapidement une sphère qui se différencie en une couche extra-embryonnaire superficielle, le périderme, puis forme, après quelques heures, une cavité centrale, semblable à une cavité blastocœlienne (présente chez la plupart des embryons de vertébrés mais normalement absente chez le poisson zèbre). Cette sphère creuse ne présente aucun mouvement cellulaire et aucune trace de différenciation de tissus ou d'organes après $24 \mathrm{~h}$ en milieu de culture (Figure 2E). À l'opposé, en présence de clones sécrétant BMP et Nodal (Figure 2F-G), un blastopore apparaît (Figure $2 H$ ). Les explants instruits par BMP et Nodal présentent des mouvements de gastrulation très similaires à ceux que l'on observe chez les gastrula d'amphibiens ainsi que la formation d'axes antéro-postérieur et dorso-ventral bien définis (Figure 21). Maintenus en milieu de culture, ces explants instruits forment in vitro des embryoïdes très similaires aux embryons sauvages, avec des organes dérivés des différents feuillets embryonnaires, une symétrie bilatérale et des polarités dorso-ventrale et antéro-postérieure claires (Figure 2J-K) [7].

\section{Conclusions et perspectives}

Notre étude démontre que l'on peut instruire des cellules pluripotentes de blastula de poisson zèbre avec des gradients de morphogènes (Nodal et BMP) et les conduire à s'organiser en un embryon complet contenant organes et tissus. Ces deux facteurs agissent en amont, au sommet de la cascade des régulations et inductions géniques qui caractérisent les programmes développementaux. Ils sont donc suffisants pour enclencher le développement embryonnaire et pour induire et réguler, directement ou indirectement, toutes les voies de signalisation requises pour achever un programme développemental complet.

Les vertébrés dérivant tous d'un ancêtre commun, nous pouvons prédire que les lois établies en utilisant un modèle animal téléostéen seront pour l'essentiel conservées chez tous les vertébrés, y compris chez l'homme. Des études complémentaires visant à reproduire avec un modèle mammifère, la souris, les résultats obtenus chez le poisson zèbre sont en cours. Ces études offrent une base de travail pour des recherches futures dans le domaine de la médecine régénérative, dont le but serait de fabriquer des tissus et organes humains in vitro à partir de populations de cellules pluripotentes en vue d'applications thérapeutiques en médecine régénérative. $\diamond$

Construction of a vertebrate embryo

from two morphogen gradients

\section{LIENS D'INTÉRÊT}

Les auteurs déclarent n'avoir aucun lien d'intérêt concernant les données publiées dans cet article.

\section{RÉFÉRENCES}

1. De Robertis EM, Kuroda H. Dorsal-ventral patterning and neural induction in Xenopus embryos. Annu Rev Cell Dev Biol $2004 ; 20: 285-308$.

2. Langdon $Y G$, Mullins MC. Maternal and zygotic control of zebrafish dorsoventral axial patterning. Annu Rev Genet $2011 ; 45: 357-77$.

3. Spemann H, Mangold H. Induction of embryonic primordia by implantation of organizers from a different species. 1923. Int J Dev Biol 2001 ; 45 : 13-38.

4. De Robertis EM. Spemann's organizer and selfregulation in amphibian embryos. Nat Rev Mol Cell Biol 2006 ; 7 : 296-302

5. Agathon A, Thisse C, Thisse B. The molecular nature of the zebrafish tail organizer. Nature 2003 ; 424 : 448-52.

6. Fauny JD, Thisse B, Thisse C. The entire zebrafish blastula-gastrula margin acts as an organizer dependent on the ratio of Nodal to BMP activity. Development 2009 ; 136 : 3811-9.

7. Xu PF, Houssin N, Ferri-Lagneau KF, et al. Construction of a vertebrate embryo from two opposing morphogen gradients. Science $2014 ; 344$ : 87-9.

8. Thisse B, Wright CV, Thisse C. Activin- and Nodalrelated factors control antero-posterior patterning of the zebrafish embryo. Nature $2000 ; 403: 425-8$.

\section{NOUVELLE}

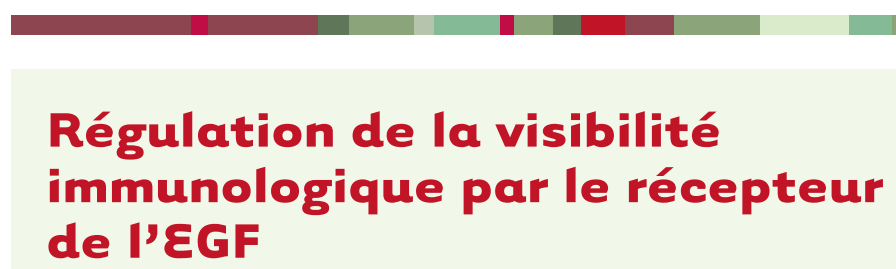

Pierre Vantourout ${ }^{1,2}$, Adrian Hayday ${ }^{1,2,3,4}$
> Le terme «visibilité immunologique» désigne l'ensemble des phénomènes permettant au système immunitaire de détecter tout statut anormal des cellules de l'organisme. Traditionnellement, ceux-ci furent principalement considérés dans le contexte des infections, comme par exemple la présentation, par les complexes majeurs d'histocompatibilité (CMH), d'antigènes du «non-soi » dérivant de protéines exprimées par des agents pathogènes. II est à présent très bien établi que le système immunitaire est également capable de détecter des dérégulations cellulaires causées par divers types de stress dits «stériles », tels

\footnotetext{
${ }^{1}$ Peter Gorer Department of Immunobiology, King's College London, London, Royaume-Uni ; ${ }^{2}$ London Research Institute, Cancer Research UK, London, Royaume-Uni.

${ }^{3}$ Medical Research Council Centre for Transplantation Biology, London, Royaume-Uni.

${ }^{4}$ Comprehensive Biomedical Research Centre of Guy's and St Thomas' Hospitals and King's College London, Great Maze Pond, SEl 9RT London, Royaume-Uni. pierre.vantourout@kcl.ac.uk adrian.hayday@kcl.ac.uk
}

que des perturbations physico-chimiques ou une transformation tumorale. Cette « réponse lymphoïde au stress » (RLS) [1] est principalement le résultat de la détection du «soi modifié » par les cellules du système immunitaire via la 